\title{
A Cartel Maintenance Framework to Enforce Cooperation in Wireless Networks with Selfish Users
}

\author{
Zhu Han, Zhu Ji, and K. J. Ray Liu
}

\begin{abstract}
In distributed wireless networks without centralized control, each user is its own authority to maximize its own performance. This distributed characteristic provides the users with the incentives of greedy competitions for the network resources such as bandwidth and transmission time. Such competitions deteriorate the system performance dramatically and result in low non-cooperative performances. Ensuring cooperation among selfish users can improve the performances and thus becomes an important issue for such wireless networks. In this paper, a Cartel Maintenance repeated game framework is proposed to enforce the cooperation among selfish users. The soul of Cartel Maintenance is to construct contracts among independent individuals for cooperative benefits and non-cooperative punishment, so as to limit inefficient competitions. In the proposed framework, a trigger-punishment game rule is designed to encourage the users to follow the cooperative strategy. First, all users agree to cooperate. Then in the following time slot, if users observe the others play cooperatively, cooperation will be played. If some users detect that others may defect based on the observed information, these users will play punishment phases for a certain period of time. This punishment is optimized so that the gain obtained by the deviation users is outweighed by the future punishments. Therefore, no user has the incentives to deviate, and the cooperation among selfish users is enforced. Then, the framework is employed to the multiple random access scenarios in wireless networks with selfish users, where the closed-form optimal solutions of cooperation enforcement are derived. The simulation results show that the proposed scheme can achieve significant performance gains over the non-cooperation scheme by having enough punishment threat to keep the cooperation among users.
\end{abstract}

Index Terms-Game theory, distributed control, and cooperative systems.

\section{INTRODUCTION}

$\mathbf{I}$ $\mathrm{N}$ RECENT years, distributed wireless networks, which consist of a collection of radio transceivers without requiring centralized administration or pre-arranged fixed network infrastructure, have been investigated intensively. Considering the application scenarios in which the users are "selfish" and act non-cooperatively to maximize their own interests, the

Manuscript received December 3, 2006; revised June 6, 2007; accepted July 11, 2007. The associate editor coordinating the review of this letter and approving it for publication was W. Zhuang. This work is partially supported by MURI AFOSR F496200210217. This work was partially presented at the IEEE Globe Telecommunications Conference 2004.

Z. Han is with the Department of Electrical and Computer Engineering, Boise State University, Boise, Idaho (e-mail: zhuhan@boisestate.edu).

Z. Ji is with Qualcomm, San Diego, CA.

K. J. R. Liu is with the Electrical and Computer Engineering Department, University of Maryland, College Park, MD.

Digital Object Identifier 10.1109/TWC.2008.061014. performances of such networks are deteriorated dramatically due to the inefficient competitions for the wireless resources among selfish users. The greediness of selfish users and the distributed network structure challenge the feasibility of the conventional approaches and require novel techniques for distributed and efficient networking. Thus, ensuring cooperation among selfish users becomes an important issue for designing wireless networks.

In distributed wireless networks, mobile users aim to selfishly maximize their own performances. Such a fact motivates the adoption of the Game Theory [1], which studies the mathematical models of conflict and cooperation among intelligent and rational decision makers. Rational means that each individual's decision-making behavior is consistent with the maximization of some subjective expected utility. Intelligent means that each individual understands everything about the structure of the situation, including the fact that others are intelligent rational decision makers. Non-cooperative game, cooperative game, and repeated game are three major components of Game Theory.

In the literature, game theory has been primarily applied to the research areas of flow control [2] and routing [3]. Recently, different types of game approaches have been introduced to several areas of wireless communications. The non-cooperation game theory was studied in [4] for power control problems, where pricing technique was used to achieve Pareto optimality. In [5], the non-cooperation game approach was employed for subcarrier-assignment in multi-cell OFDMA systems. The non-cooperative game theory has also been studied for self-organizing mobile ad-hoc wireless networks (MANET). In [6], the reputation-based game approaches were proposed to encourage packet-forwarding among users. In [7], the authors proposed a non-cooperative approach for encouraging collaboration in MANET. In regard to the cooperation game theory, in [8], an cooperative game approach named Nash Bargaining Solution was studied in the scenario of power, rate, and subcarrier allocation for single-cell OFDMA systems to have a fair and efficient performance. Besides, in the repeated game, the behaviors of users at multiple stages are studied to dynamically optimize the wireless resource allocation over time. In [9], the repeated game approach was proposed for the optimal routing control. In [10], the authors used the Markov chain theory to model the multiple-access problem as a repeated game with perfect information. The packet forwarding schemes using "TIT for TAT" schemes 
were proposed in [11], [12]. Auction theory, one of the most important applications of Game Theory, has also been applied to MANETs to provide incentives for users to cooperate such as [13], where VCG auction was adopted to encourage truthtelling and discover the most cost-efficient routes in ad hoc networks. Some preliminary result of this paper is presented in [14].

In this paper, we incorporate the repeated game theory to study how to enforce cooperations among distributed selfish users in wireless networks. First, inspired by the microeconomy approach in [15], we propose a trigger-punishment Cartel-Maintenance framework to enforce cooperation among users. The soul of Cartel Maintenance is to construct contracts among independent individuals for cooperative benefits and non-cooperative punishment, so as to limit inefficient competitions. We assume that the rational users optimize their profits over time. At first, the users agree to operate at some cooperation points. Meanwhile, the users monitor their performances distributively. If the performances are lower than certain trigger thresholds, it probably means that some users act greedily by deviating from the agreed cooperation point. The deviation benefits the greedy users but impairs the performances of the other users who still act cooperatively. In this situation, the other users will play non-cooperatively to compete for more resources for a certain period of time. Consequently, the performances of the users including the deviating users drop dramatically. So the current gain of the deviation is outweighed by the loss due to the future punishments from the others. As a result, all users have no incentive to deviate from the agreed cooperation point and are enforced to cooperate in a distributed manner by the above Cartel-Maintenance repeated-game framework.

We consider the distributive rate control scenario in wireless networks in which several distributed and greedy users share an access point via some multiple-access protocol to communicate with a remote destination. The scenario fits a variety of practical situations such as ad hoc networks and sensor networks. The proposed framework is employed to the above scenario to enforce the cooperation and enhance the system performance. For the distributed users in the network, there are costs to transmit their packets, and also benefits if their packets are successfully transmitted. Each user's profit is defined as the benefits minus the costs. The users are able to adapt their packet transmission rates for the cooperation play or punishment play. They can locally observe their successful packet transmission probabilities, and correspondingly play cooperation or non-cooperation. Based on a close approximation of the objective function, we derive the optimal parameters of the packet transmission rate, punishment time, and trigger threshold for the distributed greedy users in closedforms. The simulations demonstrate that the proposed scheme can enforce the selfish users to cooperate and have an efficient system outcome using the optimal punishment threat. The resulting performances of the proposed distributed scheme are close to those of the centralized-control scheme and much better than those of the non-cooperative scheme.

This paper is organized as follows: In Section II, we provide a brief introduction of repeated game theory and then present the trigger-punishment Cartel Maintenance framework. In Sec- tion III, we provide the system model of a wireless network scenario. In Section IV, we employ the proposed framework to this wireless network and derive the corresponding optimal parameters. In Section $\mathrm{V}$, simulation results are provided. Conclusion is drawn in Section VI.

\section{The Cartel Maintenance Repeated Game FRAMEWORK}

In this section, we first discuss the basic concepts of the repeated game theory. Then, the Cartel Maintenance repeated game framework with the trigger punishment game rule is designed to enforce cooperations among distributed selfish users.

\section{A. Repeated Game Theory Basics}

A game $G$ in the strategic form has three elements: the set of users (players) $i \in \mathcal{I}$, which is a finite set $\{1,2, \ldots, N\}$; the strategy space $\mathcal{S}_{i}$ for each user $i$; and utility functions $\pi_{i}$, which measures the outcome of the $i$ th user for each strategy profile $\sigma=\left(\sigma_{1}, \sigma_{2}, \ldots, \sigma_{N}\right)$, where user $i$ controls its strategy $\sigma_{i} \in \mathcal{S}_{i}$. We define $\sigma_{-i}$ as the strategies of user $i$ 's opponents, i.e., $\sigma_{-i}=\left(\sigma_{1}, \ldots, \sigma_{i-1}, \sigma_{i+1}, \ldots, \sigma_{N}\right)$. In static games, the interaction between users occurs only once, while in dynamic games the interaction occurs several times and may continue forever.

To analyze the outcome of a game, the Nash Equilibrium is a well-known concept, which states that in the equilibrium every agent selects a utility-maximizing strategy given the strategies of other agents. A strategy profile $\sigma^{*}$ is a Nash Equilibrium if, for all users,

$$
\pi_{i}\left(\sigma_{i}^{*}, \sigma_{-i}^{*}\right) \geq \pi_{i}\left(\sigma_{i}, \sigma_{-i}^{*}\right), \forall \sigma_{i} \in S_{i},
$$

i.e., a Nash equilibrium is a profile of strategies such that each user's strategy is an optimal response to the other users' strategies, and $\sigma^{*}$ is called the Nash Equilibrium Point (NEP). The importance of an NEP is that it is a point where no noncooperative user has an incentive to deviate.

However, one problem with an NEP is that it is not necessarily very efficient in performances. If the users play cooperatively, the performances can be greatly improved. Thus, the question arises that how to enforce the greedy users to cooperate with each other. The repeated game provides us possible mechanisms to enforce the users to cooperate by considering long-term scenarios. In the repeated games, the users face the same static game in every period, and the user's overall payoff is a weighted average of the payoffs in each stage over time. In the repeated game, the users can observe some information reflecting their opponents' past play. Hence, they are able to condition their future plays on the observed information in history to obtain better equilibria. The information revealed by the game outcome can be observed by all users and is named as the public information.

Definition 1: Let $G$ be a static game and $\beta$ be a positive discount factor which is less than 1 . The $\mathcal{T}$-period repeated game, denoted as $G(\mathcal{T}, \beta)$, consists of game $G$ repeated $\mathcal{T}$ times. The game payoff is given by

$$
V_{i}=\sum_{t=1}^{\mathcal{T}} \beta^{t-1} \pi_{i}^{t},
$$


where $\pi_{i}^{t}$ denotes the payoff to user $i$ in period $t$. If $\mathcal{T}$ goes infinity, then $G(\infty, \beta)$ is referred as the infinitely-repeated game. In this paper, we employ the infinitely-repeated game. In fact, the repeated game may not be necessarily infinite as long as the users do not know when the game ends. For the simplicity of notation and presentation, we will derive our scheme based on the infinitely repeated game.

In many situations, the users are only able to obtain imperfect information of the strategies of their opponents based on the public information. Moreover, at stage $t$, each user not only knows the history of the public information $h^{t}$ but also knows the private histories of its own past actions, $z_{i}^{t}$. We study the situation where users ignore their private information in choosing their actions. This situation leads to the public strategies as follows.

Definition 2: Strategy $\sigma_{i}$ is a public strategy if it only depends on the public history of the game and is not affected by the users' private histories, i.e., $\sigma_{i}^{t}\left(h^{t}, z_{i}^{t}\right)=\sigma_{i}^{t}\left(h^{t}, \hat{z}_{i}^{t}\right)$ for all periods $t$, public histories $h^{t}$, and different private histories $z_{i}^{t}$ and $\hat{z}_{i}^{t}$.

Then, it is worth studying whether a public strategy induces a Nash equilibrium from period $t$ on.

Definition 3: a repeated-game strategy $\sigma_{i}$ is a perfect public equilibrium (PPE) if: (a) Strategy $\sigma_{i}$ is a public strategy; (b) For each period $t$ and history $h^{t}$, strategy $\sigma_{i}$ yields a Nash equilibrium from that period on.

Based on the above definitions, we can see that perfect public equilibria are the equilibria that no user has incentives to deviate from in the repeated game with the public information. Because when all users use public strategies, they agree on the subsequent possible actions and outcomes given the public history $h^{t}$. The PPE further specifies the strategies having a Nash equilibrium from period $t$ on, thus eliminates the deviation incentives of rational users in the repeated game based on the concept of Nash equilibrium. Note that PPE can also be considered as an extension of the idea of subgame perfection in the repeated game [1].

Now the question is whether or not the cooperation among users can be enforced by the repeated games to generate better performances. From Folk Theorem [1] below, we know that in an infinitely repeated game, any feasible outcome that gives each player better payoff than the Nash equilibrium can be obtained.

Theorem 1: Folk Theorem: Let $\left(\pi_{1}, \ldots, \pi_{N}\right)$ be the payoffs from a Nash equilibrium of game $G$ and let $\left(V_{1}, \ldots, V_{N}\right)$ be any feasible payoffs from $G$. If $V_{i}>\pi_{i}$ for every player $i$, then there exists an equilibrium of $G(\infty, \beta)$ that attains $\left(V_{1}, \ldots, V_{N}\right)$ as the average payoff, provided that $\beta$ is sufficiently close to 1 .

This equilibrium can have better performances than those of static game NEP. However, the Folk's Theorem does not explain how to enforce the equilibrium. In the following subsection, we further develop the detailed game rule for enforcing the cooperation among users to achieve this better equilibrium.

\section{B. Cartel Maintenance Framework with Trigger Punishment Game Rule}

The basic idea for the proposed Cartel Maintenance repeated game framework is to provide enough threat to greedy users so as to prevent them from deviating from cooperation. First, the cooperative point is obtained so that all users have better performances than those of non-cooperative NEPs. However, if any user deviates from cooperations while the others still play cooperatively, this deviating user has a better utility, while the others have relatively worse utilities. If no rule is enforced, the cooperative users also have incentives to deviate. Consequently, the network deteriorates to the noncooperation results with inefficient performances. The proposed framework provides a mechanism so that the current defecting gains of the selfish user will be outweighed by the future punishment from the other users. For any rational user, this threat of punishment prevents them from deviation. As a result, the cooperation is enforced.

The proposed trigger rule is a strategy to introduce punishment on the defecting users. In the trigger rule, the users start with cooperation. Assume each user can observe the public information (e.g. the outcome of the game), $P_{t}$ at time $t$. Examples of this public information can be the successful transmission rate and network throughput. Notice that such public information is mostly imperfect or simply partial information about the users' strategies, which is because there is limited ability for signaling all information in practice. Here we assume a larger $P_{t}$ stands for a higher cooperation level, resulting in higher performances for all users. Let the cooperation strategies be $\bar{\lambda}=\left[\lambda_{1}, \lambda_{2}, \ldots, \lambda_{N}\right]^{\top}$ and the noncooperative strategies be $\bar{s}=\left[s_{1}, s_{2}, \ldots, s_{N}\right]^{\top}$, respectively. The trigger-punishment game rule is characterized by three parameters: the optimal punishment time $T$, trigger threshold $P^{*}$, and the cooperation strategy $\bar{\lambda}$. The trigger punishment strategy $\left(\bar{\lambda}, P^{*}, T\right)$ for distributed user $i$ is given as follows:

(a) User $i$ plays the strategy of the cooperation phase, $\bar{\lambda}$, in period 0 ;

(b) If the cooperation phase is played in period $t$ and $P_{t}>$ $P^{*}$, user $i$ plays the cooperation phase in period $t+1$;

(c) If the cooperation phase is played in period $t$ and $P_{t}<$ $P^{*}$, user $i$ switches to a punishment phase for $T-1$ periods, in which the users play a static Nash equilibrium $\bar{s}$ regardless of the realized outcomes. At the $T$ th period, play returns to the cooperative phase.

Note that $\bar{s}$ generates the non-cooperation outcome, which is much worse than that generated by the cooperation strategy $\bar{\lambda}$. Therefore, the deviating selfish user has much lower utilities in the punishment phase. Moreover, the punishment time $T$ is designed to be long enough to let all cheating gains of the selfish users be outweighed by the punishment. So the users have no incentive to deviate from cooperation, since the users aim to maximize the long-run payoffs over time. Moreover, we assume the users are synchronized in the sense that they can start punishment and restore cooperation simultaneously. This assumption fits the situations where the network topology is relatively simple and the public information can be easily observed. If the synchronization assumption is not valid, a certain period of time is required for transition from the 


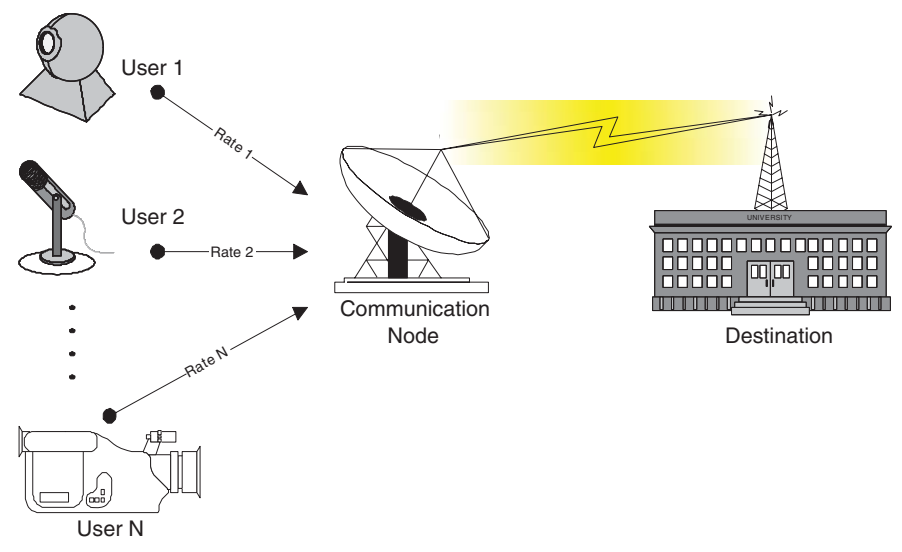

Fig. 1. Illustration of a wireless network with selfish users.

punishment phase back to the cooperative phase.

The rest of the problem is how to calculate the optimal parameters of $\left(\bar{\lambda}, P^{*}, T\right)$, i.e., how to construct a Cartel so that the benefit is optimized and the incentive for deviation is eliminated. We define $\mathcal{P}_{\text {trig }}=\operatorname{Pr}\left(P_{t}<P^{*}\right)$, which is the trigger probability that the realization of public information is less than the trigger threshold. If we discuss the different future situations in (2), the expected payoff $V_{i}$ is given as follows:

$$
\begin{array}{r}
V_{i}\left(\bar{\lambda}, P^{*}, T\right)=\pi_{i}(\bar{\lambda})+\left(1-\mathcal{P}_{\text {trig }}\right) \beta V_{i}\left(\bar{\lambda}, P^{*}, T\right) \\
\quad+\mathcal{P}_{\text {trig }}\left[\sum_{t=1}^{T-1} \beta^{t} \pi_{i}(\bar{s})+\beta^{T} V_{i}\left(\bar{\lambda}, P^{*}, T\right)\right], \forall i
\end{array}
$$

where $\pi_{i}(\bar{\lambda})$ and $\pi_{i}(\bar{s})$ are the cooperation and noncooperation payoffs, respectively. The first term at the right hand side of (3) is the current expected payoff if cooperation is played; the second term and third term are the payoffs for two different results depending on whether or not the punishment is triggered, respectively. Notice that $V_{i}$ is a function of not only users' strategies $\bar{\lambda}$ but also the game parameters $P^{*}$ and $T$. Our objective is to maximize the expected payoff $V_{i}$ for each user while the optimal strategy yields an NEP for the proposed algorithm. In order to achieve the NEP given $P^{*}$ and $T$, the optimal strategies of the repeated games can also be characterized by the first-order necessary conditions,

$$
\frac{\partial V_{i}\left(\lambda_{i}, \lambda_{-i}\right)}{\partial \lambda_{i}}=0, \forall i .^{1}
$$

If all users have the same utility and the game outcome is symmetric for all users, the solution $\lambda^{*}$ of the first-order conditions is the same for all users. This solution is also a function of parameter $P^{*}$ and $T$. In order to obtain the optimal $P^{*}$ and $T$ for maximizing the expected payoff $V_{i}$, we have the following differential equations

$$
\frac{\partial V_{i}\left(P^{*}, T\right)}{\partial P^{*}}=0, \text { and } \frac{\partial V_{i}\left(P^{*}, T\right)}{\partial T}=0, \forall i .
$$

In general, (4) and (5) need to be solved via numerical methods. For a certain structure of the payoff function, we are able to derive the closed-form optimal configuration $\left\{\bar{\lambda}, P^{*}, T\right\}$.

\footnotetext{
${ }^{1}$ Notice that the optimal strategies might not be the strategies obtained from centralized control.
}

In the following sections, based on the above discussion, we will employ the repeated game framework to a specific wireless network, develop efficient and distributed rate control strategies, and further derive the optimal closed-form configuration using an approximation.

\section{System Model}

Figure 1 shows the block diagram of a multiuser wireless network. There are many distributed users and one access point. Each user can transmit its data packets to the access point by using the multiple access protocols such as Aloha and carrier sense multiple access (CSMA). Each user can adjust its packet transmission rate to the access point. The access point has the ability to transmit the data packets to the remote destination via a wireless link. The scenario can be viewed as a snapshot of a clustered ad hoc network. The access point is the cluster head, which is responsible for forwarding packets for their nearby users to the other cluster heads. The network scenario also fits the sensor network scenario, where a powerful node collects information from different autonomous sensors and forwards it to remote stations. Assume the feedback mechanism such as ARQ exists between the access point and the destination, so that the successful packet transmission probabilities can be obtained. Since there is no central authority in the above networks, each user is its own authority and inclines to act greedily to compete for the network resources by having a high packet transmission rate, which results in a lot of collisions of the multiple-access protocols and consequently inefficient performances. In the rest of this section, we model the utility of profit reflecting the interests of selfish users.

Assume there are $N$ active users in the wireless networks. The transmission times for the data packets are divided into time slots. The packet arrival can be modeled as the Poisson distribution. Users can adjust their strategies by changing the arrival rates. The packet transmission rate vector for all users is denoted by $\bar{\lambda}=\left[\lambda_{1}, \ldots, \lambda_{N}\right]^{\top}$, where $\lambda_{i}$ is the rate of user $i$. Total arriving rate at the access point is then $\Lambda=\sum_{i=1}^{N} \lambda_{i}$. Each user intends to increase its transmission rate. However, arbitrary increases of the packet transmission rates result in a higher probability of collision at the access point and reduce the system throughput. In addition, the probability of successful transmission is also affected by the link quality from the access point to the destination. All users can observe the successful packet-transmission probability that functions as the public information. Note that this public information can be announced using trivial signalling with negligible costs. The probability can be expressed as $\hat{P}=\theta \cdot P(\Lambda)$, where function $P: \Re_{+} \rightarrow \Re_{+}$denotes the successful transmission probability per packet that is a function of $\Lambda$ for the multiple-access protocol, and $\theta$ is the probability of successful transmissions from the access point to the destination that is modeled as an identically and independently distributed sequence of random variables with mean $\mu$, probability density function (PDF) $f$, and cumulative distribution function (CDF) $F$.

In general, the successful packet transmission probability $P(\Lambda)$ is not a linear function. Within a typical working range, we can approximate $P$ as a linear and decreasing function of 


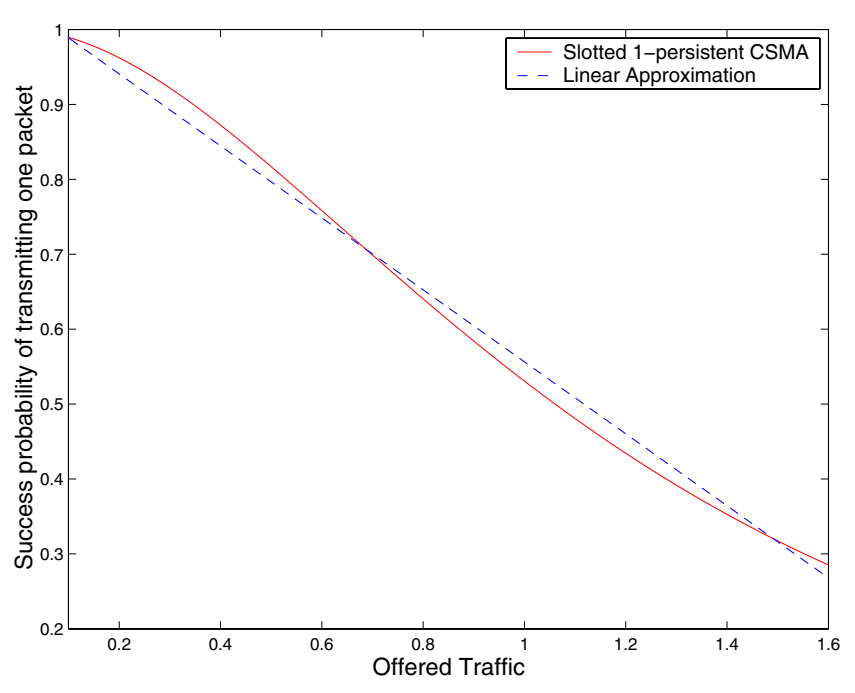

Fig. 2. Linear approximation.

total packet transmission rate $\Lambda$ as

$$
P(\Lambda) \approx a-b \Lambda
$$

where $a$ and $b$ are positive constants. From the simulations shown later, (6) is a good approximation as shown in Figure 2. Such a linear approximation allows us to obtain a closed-form analytical solution that provides insights on the formulated problem in the next section. Other nonlinear models with better accuracy can also be employed using a similar analysis. However, the closed-form solution might not be able to be obtained and numerical methods can be utilized to find the solutions in a straightforward way.

We assume a benefit of $c_{2}$ can be obtained for each successful transmitted packet. Thus, the user's benefit can be written as

$$
u_{i}(\bar{\lambda})=c_{2} \hat{P}(\Lambda) \lambda_{i}=c_{2} \theta \cdot(a-b \Lambda) \lambda_{i} .
$$

Without loss of generality, we assume $c_{2}=1$.

Because of power consumption or pricing, there exists a cost for each packet transmission. In this paper, we use linear model for the cost as:

$$
C=c_{0}+c_{1} \lambda_{i}
$$

where $c_{0}$ is the basic cost to maintain the link and $c_{1}$ is the cost per transmission packet.

Considering the benefit and cost simultaneously, each user's profit is defined as the benefit minus the cost, which can be written as

$$
\pi_{i}(\bar{\lambda})=\hat{P} \lambda_{i}-C=\theta(a-b \Lambda) \lambda_{i}-c_{0}-c_{1} \lambda_{i}, \forall i
$$

Define $\Lambda_{-i}=\Lambda-\lambda_{i}=\sum_{j \neq i} \lambda_{j}$ as the total transmission packet rate of the other users. The $i$ th user's expected profit in (9) can be further represented as a function of $\lambda_{i}$

$$
\pi_{i}(\bar{\lambda})=\left[A-B\left(\Lambda_{-i}+\lambda_{i}\right)\right] \lambda_{i}-c_{0},
$$

where $A=\mu a-c_{1}$ and $B=\mu b$. Remember that $\mu$ is the mean of $\theta$.

\section{EnForce CoOperation for Multiple ACCESS IN WIRELESS NETWORKS}

In this section, we first present the motivations of the proposed strategy by analyzing both the performances of the non-cooperative scheme and the scheme with fully centralized control. Then the optimal parameters of our scheme are deduced in closed forms.

In order to understand the motivations of introducing the repeated game approach, we study the users' behavior in the static non-cooperative game. In this non-cooperative game, the users act selfishly and independently to increase their profits by adapting their packet transmission rates, i.e.,

$$
\arg \max _{\lambda_{i}} \pi_{i}, \forall i
$$

Let $\bar{s}=\left[s_{1}, \ldots, s_{N}\right]^{\top}$ denote the optimal rate vector for the above non-cooperative optimization. By taking the derivatives of $\lambda_{i}$ in (9) for all users and solving the equations, we have

$$
s_{i}=s=\frac{A}{B(N+1)}, \forall i .
$$

By substituting (12) into (10), the non-cooperation profit is

$$
\pi_{i}(\bar{s})=\frac{A^{2}}{B(N+1)^{2}}-c_{0}, \forall i .
$$

We assume that

$$
0<c_{1}<\mu a
$$

and

$$
0<c_{0}<\frac{\left(\mu a-c_{1}\right)^{2}}{\mu b(N+1)^{2}} .
$$

Both (14) and $b>0$ imply that $A$ and $B$ are positive constants. Notice that (15) guarantees that users earn positive profits, i.e., $\pi_{i}(\bar{s})>0, \forall i$.

On the other hand, if there exists centralized control, the system has complete information about all users' strategies and can maximize the system overall profit as

$$
\arg \max _{\bar{\lambda}} \sum_{i=1}^{N} \pi_{i} .
$$

Denote users' rate vector which maximizes the expected overall profit by $\bar{r}=\left[r_{1}, \ldots, r_{N}\right]^{\top}$. Substituting (9) in (16) and solving the first-order conditions of (16), we have the solutions as

$$
r_{i}=r=\frac{A}{2 B N}, \forall i
$$

Substituting (17) into (10), the centralized controlled profit is

$$
\pi_{i}(\bar{r})=\frac{A^{2}}{4 B N}-c_{0}, \forall i .
$$

From (12) and (17), we observe that the non-cooperative packet transmission rate $\bar{s}$ is much higher than the centralized one $\bar{r}$ due to the selfishness. As a result, from (13) and (18), we can see that, as long as there is more than one user, each user's expected profit with centralized control is higher than that of the non-cooperative results.

Further, the non-cooperative strategy $\bar{s}$ is a static Nash equilibrium, which implies that no user has the deviation incentive. On the other hand, although centralized strategy 
$\bar{r}$ achieves much better outcomes, it provides the selfish users' deviation incentives. If user $i$ deviates from (17) by transmitting at a higher rate, while others' transmission rates remain the same, from (10) and (18) this deviating user gets the profit

$$
\pi_{i}=\left\{A-B\left[\frac{(N-1) A}{2 B N}+\lambda_{i}\right]\right\} \lambda_{i}-c_{0} .
$$

By differentiating $\lambda_{i}$, if $N>1$, we have

$$
\left.\frac{\partial \pi_{i}}{\partial \lambda_{i}}\right|_{\bar{r}}=\frac{(N-1) A}{2 N}>0
$$

Thus, the deviating user can have a higher profit. However, the greediness of users will make them all deviate and eventually lead to the non-cooperative scenario. Since it is difficult and costly to implement the centralized control in many network settings, our goal is to construct a distributed rate control mechanism for wireless networks to enforce the users' cooperation with each other and achieve a better performance. The Cartel Maintenance repeated game framework discussed in the previous sections can be exploited to solve the above problem by introducing threat of punishment on non-cooperation behaviors.

Specifically, the users' expected profit is obtained by putting the profit for one stage indicated by (9) into (2). For the system model discussed in the previous section, by using the proposed framework, we then use the trigger-punishment game rule to derive the optimal solution for cooperation enforcement. At the beginning, all users are in a cooperative period with cooperative rate $\bar{\lambda}$. This can be calculated by letting the access point announce some information such as the number of users once. Then they locally monitor the realization of successful transmission probability $\hat{P}_{t}$ at time $t$. If the probability is higher than some threshold $P^{*}$, it means that all users probably transmit at the cooperative operating rate. On the other hand, if the probability drops lower than the threshold, it means that some users may cheat by transmitting at a higher rate. Consequently, the other users will play punishment by transmitting non-cooperatively according to (12) for a period of $T-1$. After the punishment period, they will come back to play the cooperation again.

It's worth mentioning that the successful transmission probability is determined by two factors: the users' transmission rates and the wireless channel condition from the access point to the destination. It is possible that all users act cooperatively but the probability is still under the threshold because of a low channel realization. Under this situation, the users will play the non-cooperative phase, because they cannot tell if the low successful transmission probability is caused by the deviations or the bad channels. This phenomenon is called "false pricing war" in the economy literature. Such phenomenon affects the system performance, which is the expense for distributed implementation. We will show this expense in the simulation results.

The remaining problem is how to find the optimal values of the cooperative rate $\bar{\lambda}$, threshold $P^{*}$ and punishment duration
$T$. From (3), the expected profit of user $i$ is given by

$$
\begin{array}{r}
V_{i}(\bar{\lambda})=\pi_{i}(\bar{\lambda})+P_{r}\left\{\hat{P} \geq P^{*}\right\} \beta V_{i}(\bar{\lambda}) \\
+P_{r}\left\{\hat{P}<P^{*}\right\}\left(\sum_{\tau=1}^{T-1} \beta^{\tau} \pi_{i}(s)+\beta^{T} V_{i}(\bar{\lambda})\right),
\end{array}
$$

where the first term at the RHS is the current expected value, the second term is the expected value at the next time slot with cooperation, and the third term is the expected value at the next time slot with non-cooperation. Then we can rewrite (21) as:

$$
V_{i}(\bar{\lambda})=\frac{\pi_{i}(s)}{1-\beta}+\frac{\pi(\bar{\lambda})-\pi(s)}{1-\beta+\left(\beta-\beta^{T}\right) F\left(P^{*} / P(\Lambda)\right)},
$$

where $F$ is CDF of $\theta$. In order to achieve the NEP, an equilibrium transmission rate vector $\bar{\lambda}^{*}$ can be achieved by solving

$$
V_{i}\left(\bar{\lambda}^{*}\right)=\max \left\{V_{i}(\bar{\lambda}) \mid \lambda_{j}=\lambda_{j}^{*} \text { for } j \neq i \text {, and } \lambda_{i} \geq 0\right\} .
$$

The above maximization can be further solved from (22) by using the first-order condition as

$$
\begin{aligned}
& \frac{\partial V_{i}(\bar{\lambda})}{\partial \lambda_{i}}=\left[1-\beta+\left(\beta-\beta^{T}\right) F\left(P^{*} / P\left(\Lambda^{*}\right)\right)\right] \pi_{i}^{i}\left(\bar{\lambda}^{*}\right)+ \\
& \left(\beta-\beta^{T}\right)\left[P^{*} P^{\prime}\left(\Lambda^{*}\right) / P\left(\Lambda^{*}\right)^{2}\right] f\left(P^{*} / P\left(\Lambda^{*}\right)\right) \\
& {\left[\pi_{i}\left(\bar{\lambda}^{*}\right)-\pi_{i}(\bar{s})\right]=0, \forall i}
\end{aligned}
$$

where $\pi_{i}^{i}(\bar{\lambda})=\partial \pi_{i}(\bar{\lambda}) / \partial \lambda_{i}, P^{\prime}(\Lambda)=d P(\Lambda) / d \Lambda$ and let $\phi^{*}=P^{*} / P\left(\Lambda^{*}\right)$. It is obvious that the optimal packet rates satisfying (24) indicates Nash equilibria for every stage in the repeated game.

Before we derive the optimal strategies, we discuss the public strategy and perfect public equilibrium first.

Proposition 1: The optimal strategy of the triggerpunishment algorithm is a public strategy.

Proof: The optimal strategy of the trigger-punishment algorithm consists of $\lambda^{*}$ in the cooperation phases and $s$ in the punishment phases for every user. In our repeated game, the public history $h^{t}$ is the observation of $\hat{P}_{t}$ in the previous stages, which can be defined as $h^{t}=\left[\hat{P}_{1}, \hat{P}_{2}, \ldots, \hat{P}_{t-1}\right]^{\top}$. The private history $z_{i}^{t}$ refers to each user's previous outcomes, i.e., $z_{i}^{t}=\left[\pi_{i}^{1}\left(\sigma_{i}^{1}\right), \pi_{i}^{2}\left(\sigma_{i}^{2}\right), \ldots, \pi_{i}^{t-1}\left(\sigma_{i}^{t-1}\right)\right]^{\top}$, where $\sigma_{i}^{\tau}$ indicates the $i$ th user's strategy at time $\tau$. From the trigger-punishment algorithm in IV.B and (22), the optimal strategy only depends on the public history $h^{t}$, i.e., $\sigma_{i}^{t}\left(h^{t}, z_{i}^{t}\right)=\sigma_{i}^{t}\left(h^{t}, \hat{z}_{i}^{t}\right)$ for all $t$. Therefore, the optimal strategy is a public strategy.

Proposition 2: The optimal strategy of the triggerpunishment algorithm is a perfect public equilibrium.

Proof: : The optimal strategy is proved to be a public strategy in the above proposition. Then, we need to show that for each period $t$ and history $h^{t}$, the optimal strategy on current stage yields a Nash equilibrium from that period on. In our problem, the optimal repeated-game strategy is to switch between the cooperation phase strategy $\lambda^{*}$ and the punishment phase strategy $s$. As $\lambda^{*}$ is the solution of (24), it is a Nash equilibrium for (21). Moreover, the punishment strategy $s$ is proved to be a Nash equilibrium in proposition 1. Thus, we conclude the optimal strategy is a perfect public equilibrium. 
Next, in order to further understand the first-order conditions in (24), we introduce the following propositions.

Proposition 3: For any strictly concave profit function, the non-cooperative output vector $\bar{s}$ is an equilibrium for any $P^{*}$ and $T$, i.e., the noncooperative outcome of a signal stage game is a Nash equilibrium.

Proof: Considering $\bar{s}$ is the optimal rate vector for the optimization problem in (11), $\pi_{i}^{i}(\bar{s})=0$ for $i=1, \ldots, N$. Taking the second derivative of $V_{i}(\bar{\lambda})$ in (22) with respect to $\lambda_{i}$ and letting $\bar{\lambda}=\bar{s}$, we obtain

$$
\frac{\partial^{2} V_{i}(\bar{s})}{\partial \lambda_{i}^{2}}=\frac{\partial^{2} \pi_{i}(\bar{s})}{\partial \lambda_{i}^{2}} /\left[1-\beta+\left(\beta-\beta^{T}\right) F\right]^{2}, \forall i
$$

From (10), we have $\partial^{2} \pi_{i}(\bar{\lambda}) / \partial \lambda_{i}^{2}<0$, which indicates the concavity of the profit function $\pi_{i}(\bar{\lambda})$. Further, the concavity of the profit function implies concavity of the value function $V_{i}(\bar{\lambda})$ at $\bar{s}$ by (25). Therefore, the non-cooperative output vector $\bar{s}$ is an equilibrium solving (23).

From the above proposition, we can see that the problems are not whether or not the equilibria exist, but whether, among the existing equilibria, there is one which yields the best profits of users. Basically, this proposition motivates the following deriving calculus. Specifically, we need to select the values of $P^{*}$ and $T$ to maximize the expected profit for each user, which in turn implicitly determine the optimal $\bar{\lambda}$ by (24).

Proposition 4: If users are homogenous, all users will produce exactly the same transmission rate at cooperative periods, i.e., under symmetry assumption, $\lambda_{i}=\lambda_{j}, \forall i, j$.

Proof: If there exist positive $\lambda_{k}^{*}$ and $\lambda_{l}^{*}$ for some $k$ and $l$, we can rewrite (24) as

$$
\alpha \pi_{i}^{i}\left(\bar{\lambda}^{*}\right)+\gamma\left[\pi_{i}\left(\bar{\lambda}^{*}\right)-\pi_{i}(\bar{s})\right]=0, \quad \text { for } i=k, l,
$$

where $\alpha=1-\beta+\left(\beta-\beta^{T}\right) F$ and $\gamma=\left(\beta-\beta^{T}\right) f P^{*} P^{\prime} / P^{2}$. Note $\alpha>0$ (since $0<\beta<1$ ) and $\gamma<0$ (since $P^{\prime}<0$ ). From (10), for $i=k, l$, we have

$$
\begin{gathered}
\pi_{i}^{i}\left(\bar{\lambda}^{*}\right)=A-B \Lambda^{*}-B \lambda_{i}^{*}, \\
\pi_{i}\left(\bar{\lambda}^{*}\right)-\pi_{i}(\bar{s})=\lambda_{i}^{*}\left(A-B \Lambda^{*}\right)-A^{2} /\left[B(N+1)^{2}\right] .
\end{gathered}
$$

By subtracting (26) for user $k$ from that of user $l$ and using (27) and (28), we are able to obtain

$$
\left[\alpha B-\gamma\left(A-B \Lambda^{*}\right)\right]\left(\lambda_{k}^{*}-\lambda_{l}^{*}\right)=0 .
$$

Since $\left(A-B \Lambda^{*}\right)>0, \alpha>0, \gamma<0$ and $\lambda_{k}^{*}$ and $\lambda_{l}^{*}$ are positive, (29) can be satisfied if and only if $\lambda_{k}^{*}=\lambda_{l}^{*}$.

From Proposition 2 which states the symmetry, we can denote the optimal transmission rate in cooperative periods for each user by $\lambda^{*}$. Then the total arriving rate $\Lambda^{*}=N \lambda^{*}$. The first-order necessary condition (24) can be rewritten as

$$
\begin{gathered}
{\left[1-\beta+\left(\beta-\beta^{T}\right) F\left(\phi^{*}\right)\right]\left[A-(N+1) B \lambda^{*}\right]=\left(\beta-\beta^{T}\right)} \\
\quad f\left(\phi^{*}\right)\left[\frac{P^{*} b}{\left(a-N b \lambda^{*}\right)^{2}}\right]\left[\lambda^{*}\left(A-N B \lambda^{*}\right)-\frac{A^{2}}{B(N+1)^{2}}\right] .
\end{gathered}
$$

Proposition 5: If an equilibrium characterized by $\bar{\lambda}^{*}, P^{*}$, and $T$ satisfies (24), $\lambda_{i} \in(s / N, s], \forall i$.

Proof: From (22), we know that $V_{i}(\bar{\lambda}) \geq V_{i}(\bar{s})$ if and only if $\pi_{i}(\bar{\lambda}) \geq \pi_{i}(\bar{s})$. Considering the symmetric linear structure, we have

$$
\lambda(A-N B \lambda)-c_{0} \geq A^{2} /\left(B(N+1)^{2}\right)-c_{0},
$$

where $\lambda_{i}=\lambda$ for $i=1, \ldots, N$. Further, from (31), we obtain the range of transmission rate as

$$
A /(N(N+1) B) \leq \lambda \leq A /((N+1) B),
$$

or

$$
s / N \leq \lambda \leq s .
$$

Moreover, it can be shown that $\pi_{i}^{i}(\bar{s} / N)>0$ and $\pi_{i}(\bar{s} / N)=$ $\pi_{i}(\bar{s})$. Thus, $V_{i}^{i}(\bar{s} / N)>0$ for all $i$. Therefore, $\bar{s} / N$ itself is not an equilibrium.

Based on the above propositions, we will develop the optimal values of $\bar{\lambda}, P^{*}$, and $T$ in the following part. The equilibrium value of user $i$ is $V_{i}\left(\bar{\lambda}\left(P^{*}, T\right) ; P^{*}, T\right)$. Since for each $P^{*}$ and $T$, from (30), we can calculate the Nash equilibrium $\bar{\lambda}^{*}\left(P^{*}, T\right)$, which is a function of the trigger price $P^{*}$ and $T$. Therefore, the optimal problem becomes

$$
\max _{P^{*}, T} V_{i}^{*}\left(P^{*}, T\right), i=1, \ldots, N,
$$

Since we assume all users have the same utility function, these optimal values are the same to all users. In order to obtain the optimal values of $P^{*}$ and $T$, the first-order necessary conditions should be satisfied as

$$
\frac{\partial V_{i}^{*}}{\partial P^{*}}=0, i=1, \ldots, N,
$$

and

$$
\frac{\partial V_{i}^{*}}{\partial T}=0, i=1, \ldots, N
$$

Considering the symmetric property and $\frac{\partial V_{i}\left(\bar{\lambda}^{*}\right)}{\partial \lambda_{i}}=0$, for $j \neq$ $i$, (35) and (36) can be written as

$$
\sum_{j=1}^{N} \frac{\partial V_{i}}{\partial \lambda_{j}} \frac{\partial \lambda_{j}^{*}}{\partial P^{*}}+\frac{\partial V_{i}}{\partial P^{*}}=(N-1) \frac{\partial V_{i}}{\partial \lambda_{j}} \frac{\partial \lambda_{j}^{*}}{\partial P^{*}}+\frac{\partial V_{i}}{\partial P^{*}}=0,
$$

and

$$
\sum_{j=1}^{N} \frac{\partial V_{i}}{\partial \lambda_{j}} \frac{\partial \lambda_{j}^{*}}{\partial T}+\frac{\partial V_{i}}{\partial T}=(N-1) \frac{\partial V_{i}}{\partial \lambda_{j}} \frac{\partial \lambda_{j}^{*}}{\partial T}+\frac{\partial V_{i}}{\partial T}=0 .
$$

Before solving (37) and (38), the following differentials can be calculated from (22) as

$$
\begin{gathered}
\frac{\partial V_{i}\left(\bar{\lambda}^{*}\right)}{\partial \lambda_{j}}=\frac{-\left(A-N B \lambda^{*}\right)}{\left[1-\beta+\left(\beta-\beta^{T}\right) F\left(\phi^{*}\right)\right]}, \quad \text { for } j \neq i \\
\frac{\partial V_{i}\left(\bar{\lambda}^{*}\right)}{\partial T}=\frac{\tilde{P}^{2}\left[A-(N+1) B \lambda^{*}\right] \beta^{T} \ln \beta F\left(\phi^{*}\right)}{\left(\beta-\beta^{T}\right) f\left[1-\beta+\left(\beta-\beta^{T}\right) F\left(\phi^{*}\right)\right] P^{*} b}, \\
\frac{\partial V_{i}\left(\bar{\lambda}^{*}\right)}{\partial P^{*}}=\frac{-\tilde{P}\left[A-(N+1) B \lambda^{*}\right]}{P^{*} b\left[1-\beta+\left(\beta-\beta^{T}\right) F\left(\phi^{*}\right)\right]}, \\
\frac{\partial \lambda^{*}}{\partial P^{*}}=\frac{\tilde{P}\left[A-(N+1) B \lambda^{*}\right]-b \triangle \eta}{P^{*} b K}
\end{gathered}
$$

and

$\frac{\partial \lambda^{*}}{\partial T}=\frac{\beta^{T} \ln \beta\left[P^{*} b \triangle f\left(\phi^{*}\right)-F\left(\phi^{*}\right) \tilde{P}^{2}\left(A-(N+1) B \lambda^{*}\right)\right]}{P^{*} f\left(\phi^{*}\right) b\left(\beta-\beta^{T}\right) K}$

where $\lambda_{j}^{*}=\lambda^{*}, \forall j, \tilde{P}=P\left(N \lambda^{*}\right)=a-N b \lambda^{*}, \triangle=\pi_{i}\left(\bar{\lambda}^{*}\right)-$ $\pi_{i}(\bar{s})$, and

$$
\eta=1+\frac{f^{\prime}\left(P^{*} / \tilde{P}\right)}{f\left(P^{*} / \tilde{P}\right)} \frac{P^{*}}{\tilde{P}},
$$


and

$K=\frac{B \triangle(N+1)}{A-(N+1) B \lambda^{*}}+\frac{b N \triangle(\eta+1)}{\tilde{P}}-(N-1)\left(A-N B \lambda^{*}\right)$.

By substituting (39), (41), and (42) into (37), we have

$$
\lambda^{*}=\frac{A}{2 N B}\left(\frac{N+\eta+(N+1) \mu a / A}{N+1+\eta}\right) \text {. }
$$

Substituting (39), (40), and (43) into (38) and using (30) and (46), we get that $P^{*}$ can be calculated by

$$
\frac{f\left(\phi^{*}\right)}{F\left(\phi^{*}\right)} \phi^{*}-\frac{f^{\prime}\left(\phi^{*}\right)}{f\left(\phi^{*}\right)} \phi^{*}=1 \text {. }
$$

Thus,

$$
\eta^{*}=\frac{f\left(\phi^{*}\right)}{F\left(\phi^{*}\right)} \phi^{*}
$$

Putting $\eta^{*}$ into (46), the optimal transmission rate for each user is

$$
\lambda^{*}=\frac{A}{2 N B}\left(\frac{N+\eta^{*}+(N+1) \mu a / A}{N+1+\eta^{*}}\right),
$$

where $\eta^{*}>0, \lambda^{*}>s / N$ from Proposition 3. Also, $\lambda^{*} \leq s$ yields $\eta^{*} \geq \eta^{0}$, where

$$
\eta^{0}=\frac{(N+1)[(N+1)(\mu a / A)-N]}{(N-1)} .
$$

If $\eta^{*}<\eta^{0}$, then $\lambda^{*}>s$, but it has been shown in (11) that the transmission packet rate $\bar{s}$ yields a better performance since it is an equilibrium. Considering $\lambda^{*}$ is a non-increasing function of $\eta^{*}$, the optimal transmission packet rate for each user is determined by

$$
\lambda^{*}= \begin{cases}\frac{A}{2 B N}\left(\frac{N+\eta^{*}+(N+1)(\mu a / A)}{N+1+\eta^{*}}\right), & \text { if } \eta^{*}>\eta^{0} \\ s, & \text { otherwise. }\end{cases}
$$

Then, we further explore the properties of the cooperation rate $\lambda^{*}$ obtained above.

Further, $\lambda^{*}$ satisfies the first-order conditions for $P^{*}$ and $T$, which ensures that our cooperation strategy is the optimal PPE based on our game rule. Finally, the optimal value of $T$, say $T^{*}$, can be calculated by substituting the values of $P^{*}$ and $\lambda^{*}$ acquired from (47) and (49) into the first-order necessary condition (30), which can be represented as follows

$$
T^{*}=\frac{1}{\ln \beta}
$$

$\ln \left\{\beta-\frac{(1-\beta)\left[A-(N+1) B \lambda^{*}\right]}{f\left(\phi^{*}\right)\left(b \phi^{*} / P\left(\lambda^{*}\right)\right) \Delta-F\left(\phi^{*}\right)\left[A-(N+1) B \lambda^{*}\right]}\right\}$

where $\Delta=\pi_{i}\left(\bar{\lambda}^{*}\right)-\pi_{i}(\bar{s})$. So the optimal parameters can be obtained by (47), (51), and (52).

\section{Simulation Results}

In this section, simulations are carried out to analyze the effectiveness of the proposed scheme. We assume the number of users is 10 if it is not specified in the simulation. The discounting factor $\beta$ is selected to ensure the Fork Theorem is held. $\beta$ has to be sufficiently large to have solutions for (47), (51), and (52). In our case, $\beta=0.9999$. The slotted 1-persistent CSMA [16][17] is employed as the multi-access protocol for distributed users to the access point. The linear approximation of the successful transmission probability per packet is shown in Figure 2. Here the ratio of the oneway propagation delay to packet transmission time is 0.01 and the approximation parameters $a$ and $b$ in (6) are 1.0368 and 0.4804 , respectively. As the figure shows, the linear approximation is good within a reasonable working range.

The wireless channel between the access point and the destination is modeled as a shadowing fading channel ${ }^{2}$. The path loss variation caused by the shadowing effects can be modeled using a log-normal variable $\ell$ [18]. Let $\ell_{d B}=$ $10 \log _{10} \ell$, where $\ell_{d B}$ is a zero-mean normal variable. We consider the quadrature phase shift keying (QPSK) modulation and employ the approximation of the corresponding bit error rate in [20]. The successful reception probability $\theta$ can be approximated as

$$
\theta=1-\kappa_{1} \exp \left(\frac{-\kappa_{2} \ell \gamma}{2^{k}-1}\right)
$$

where $\kappa_{1}$ and $\kappa_{2}$ are 0.2 and 1.6, respectively, $\gamma$ is the signal to noise ratio (SNR) and $k=2$, indicating QPSK modulation. Further, (53) can be rewritten as

$$
\theta=1-\kappa_{1} \exp \left(\frac{-\kappa_{2} e^{\frac{\ln 10}{10} \cdot \ell_{d B}} \gamma}{2^{k}-1}\right),
$$

We can see from (54) that $\theta$ is a function of another random variable $\ell_{d B}$. Considering $\ell_{d B}$ is a zero-mean normal variable, the distribution $f$ of $\theta$ can be obtained as [19]

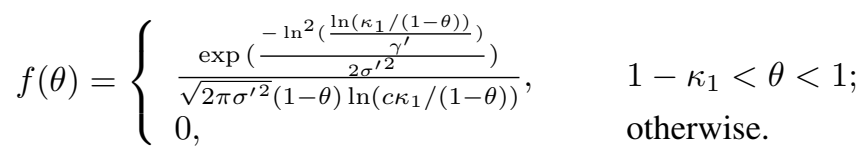

where $\gamma^{\prime}=\frac{\kappa_{2} \cdot \gamma}{2^{k}-1}$ and ${\sigma^{\prime}}^{2}=\left(\frac{\ln 10}{10}\right)^{2} \sigma^{2}$. The corresponding $\mathrm{CDF} F$ and first derivative of PDF are shown as follows.

$$
F(\theta)= \begin{cases}0, & \theta \leq 1-\kappa_{1} \\ 1-G\left(\frac{\ln \left(\ln \left(\kappa_{1} /(1-\theta)\right) / \gamma^{\prime}\right)}{\sigma^{\prime}}\right), & 1-\kappa_{1}<\theta<1,\end{cases}
$$

and

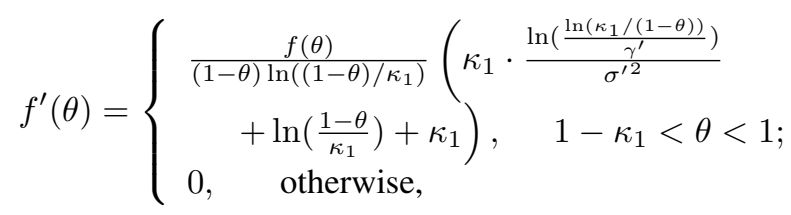

where function $G(x)$ is the CDF function of a zero-mean normal variable with unit variance.

In Figure 3, we compare the overall profits of the noncooperation scheme, centralized scheme, and repeated game scheme versus different variances of the log-normal shadowing. The performance is evaluated for two SNR values, $15 \mathrm{~dB}$ and $10 \mathrm{~dB}$, respectively. Here $c_{0}=0.001$ and $c_{1}=0.2$. It can be seen from Figure 3 that the proposed repeated game scheme can achieve much better performance than the noncooperative scheme. As the log-normal variance increases, the performance of the proposed scheme decreases accordingly. It is because the successful transmission probability depends not only on the system loads but also on the variations of

\footnotetext{
${ }^{2}$ Other fading models can be studied in a similar way
} 


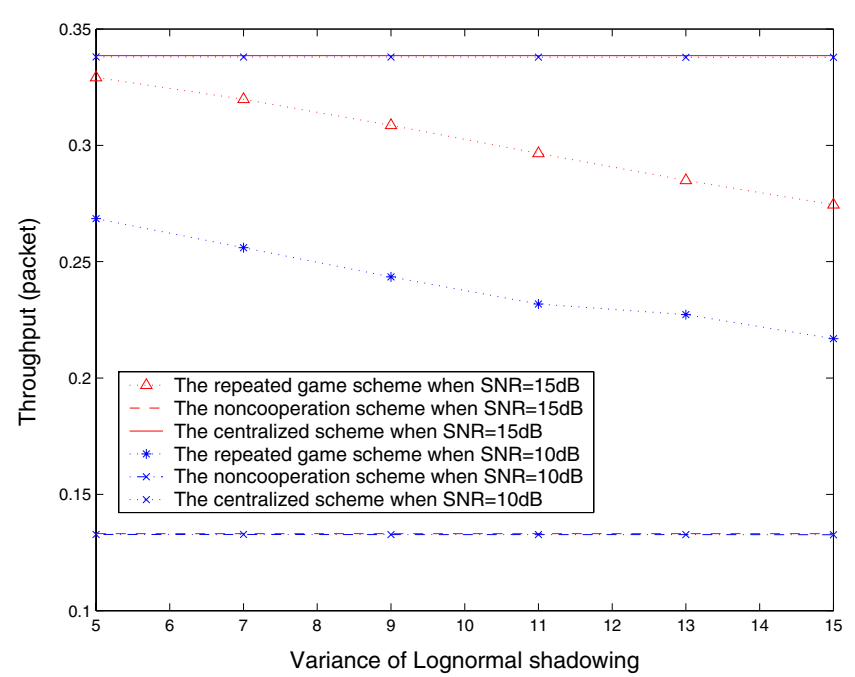

Fig. 3. System throughput of the non-cooperation, centralized, and repeated game schemes.

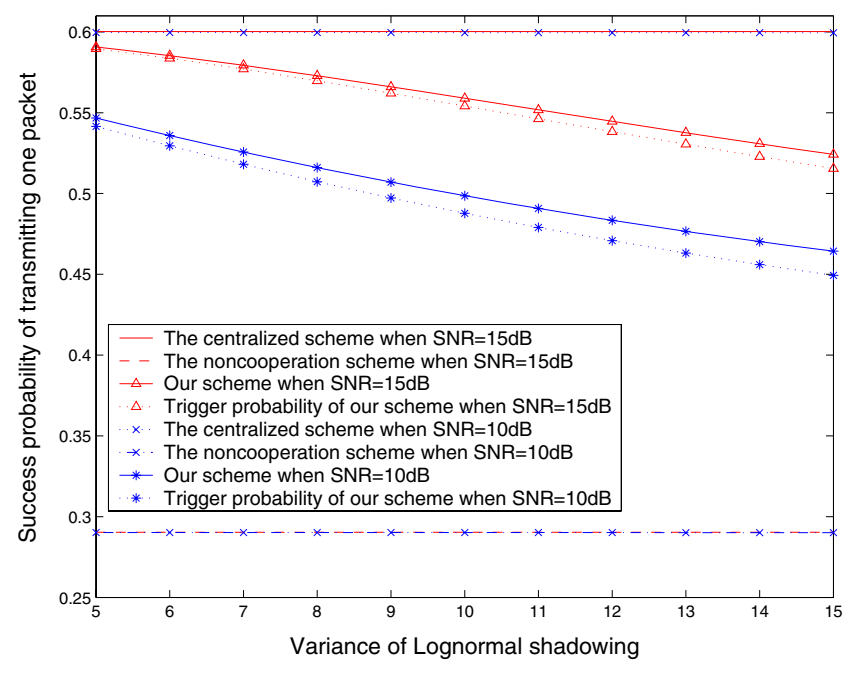

Fig. 4. Successful probability and trigger probability for different schemes.

channels. As the users act only according to their observations of the successful probabilities, they are not able to distinguish these two factors of the competitions among users or the channel variations. Consequently, they will consider a low realization of channel gain as a result of possible cheating actions of the other users and employ punishment. The performance loss compared to the centralized scheme is the expense for the distributed implementation, while there are still large performance gains compared to the non-cooperative scheme. Noting that the mean value of $\theta$ changes slightly with the variance of log-normal shadowing, the expected profit of non-cooperative and centralized schemes remains almost unchanged. Considering the channel variations also depend on the SNR at the receiving destination, we show the system throughput for different SNR values. The proposed scheme achieves higher throughput for higher SNR values, since the successful transmission probability is high when SNR is high.

In Figure 4, we compare the successful probability of transmitting per packet for different schemes. We also show the trigger threshold of the successful probability. Note that the

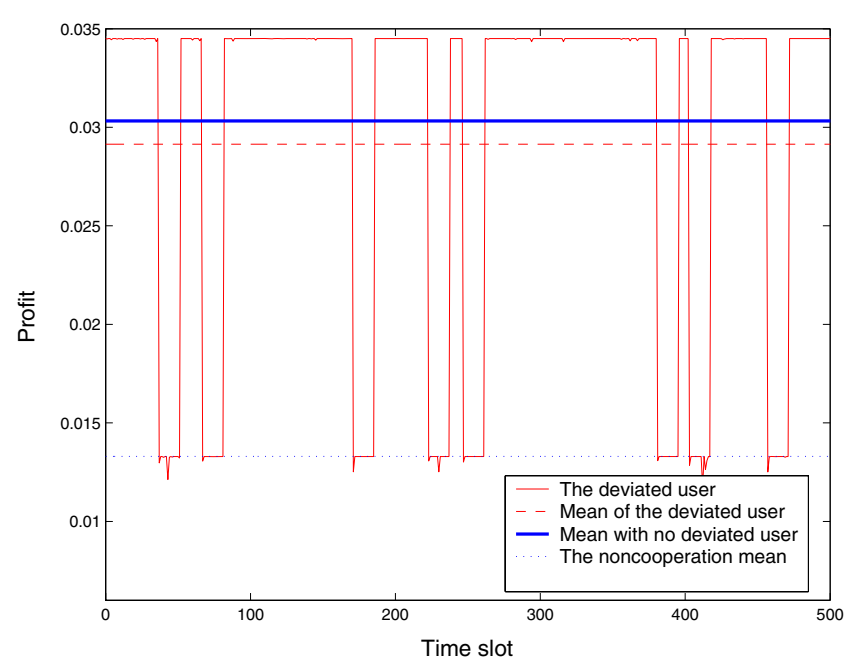

Fig. 5. Punishment for deviation.

simulation setups of this figure are the same as those of Figure 3. We can see from Figure 4 that larger channel variations result in a lower trigger threshold and a larger gap between the trigger threshold and the successful probability, which indicates that the users need to tolerate certain variations of their observations to prevent over-punishment as the channel variations increase.

In Figure 5, the punishment procedure of our scheme is shown to enforce the greedy user to cooperate. Here the variance of the log-normal shadowing is $10 \mathrm{~dB}$ and the SNR is $15 \mathrm{~dB}$. Let $c_{0}$ and $c_{1}$ be 0.001 and 0.2 , respectively. We assume one user deviates from the optimal $\lambda^{*}$ and transmits at a higher packet rate $\lambda^{\epsilon}$, while the others transmit at $\lambda^{*}$. We show the profit of this deviating user over time. For comparison, we also show the average profits when no user deviates and when all users play non-cooperation. Considering the cheating user can also observe the successful transmission probability, it will transmit at $s$ if others play punishment. We can see that the deviated user's utility, which corresponds to the top of the bars, is greater than that of the proposed scheme. However, punishment phases will be triggered due to the cheating behavior. Thus, the deviated user's current cheating gain will soon be outweighed by future punishments. The utility of the deviated user during punishment period corresponds to the bottom of the bars. In a long-run scenario, the average profit without deviating user is greater than that with the deviating user. Such facts deprive the greedy user of the cheating incentive. Consequently, the greedy users are enforced to cooperate with each other by the punishment threat. It is worthy mentioning that the utility of the punishing users during the punishment phase is low. But this short term utility loss can ensure no deviation. As a result, the utility in the cooperative phase can be enhanced, and

Considering the cost of transmission, Figure 6 shows the overall profit as a function of the cost coefficient $c_{1}$. Here, the received SNR and $\sigma^{2}$ are $15 \mathrm{~dB}$ and $10 \mathrm{~dB}$, respectively. Note that $c_{1}$ indicates the unit cost of transmitting each packet and let $c_{0}$ be 0.001 . By employing larger $c_{1}$, the users intend to transmit at a lower rate to reduce their costs and hence maximize their profits. We can see that our proposed 


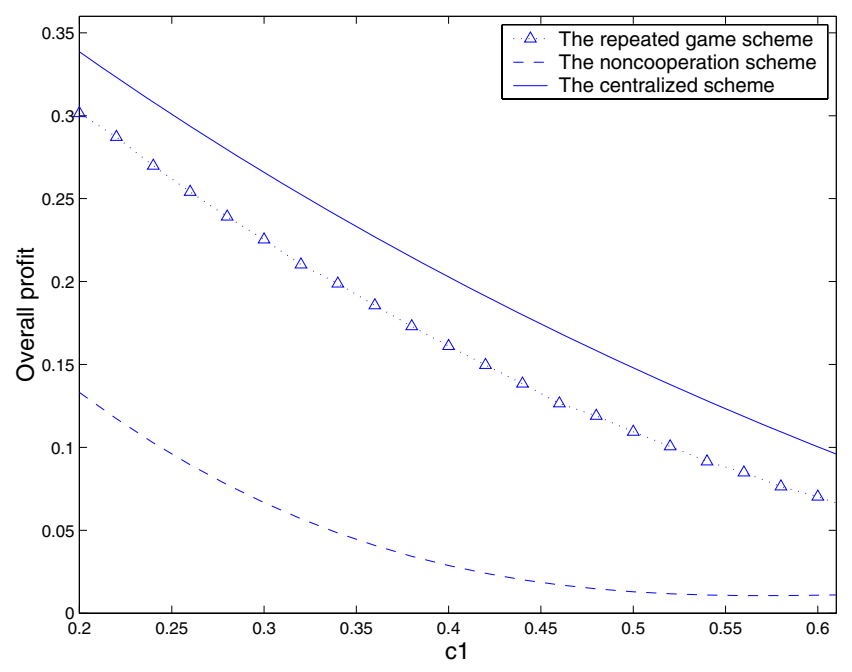

Fig. 6. Overall profit as a function of the cost coefficient $c_{1}$.

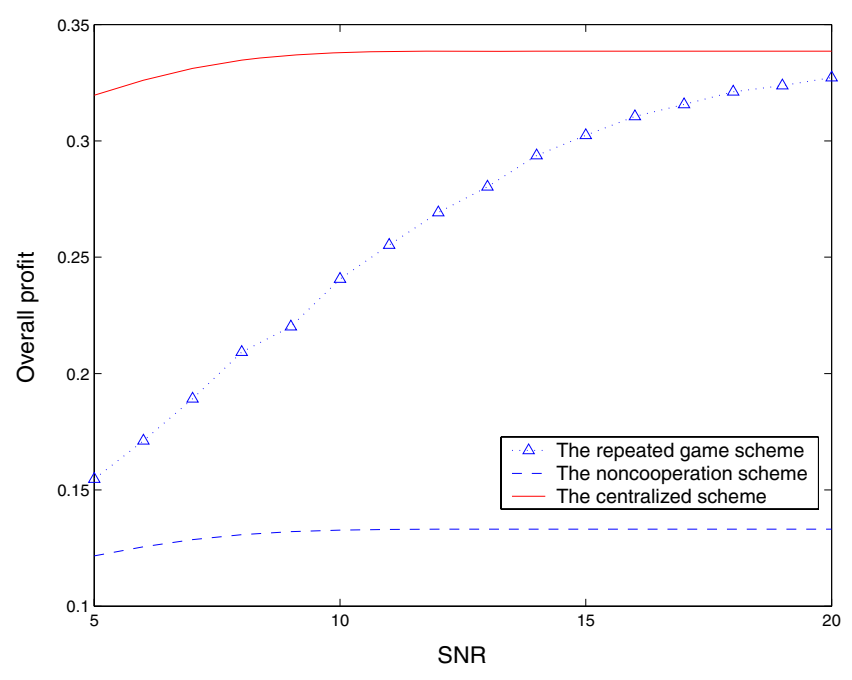

Fig. 7. Overall profit as a function of the received SNR.

scheme achieves much higher profits than the non-cooperation scheme for different cost coefficients and performs close to the centralized scheme.

In Figure 7, we show the overall profit as a function of the received SNR. Here, $c_{1}$ and $\sigma^{2}$ are set as 0.2 and $10 \mathrm{~dB}$, respectively. Since the mean value of $\theta$ increases with the increment of the received SNR, the overall profits of the non-cooperation and centralized schemes become higher accordingly. As for the repeated game scheme, there are fewer punishments triggered due to the low realizations of the log-normal shadowing (false pricing war) for higher SNR's. Hence, the performance of the proposed scheme approaches that of the centralized scheme with the increase of the received SNR.

In Figure 8, the overall profit is shown as a function of the number of users. Note that $c_{1}, \sigma^{2}$ and SNR are 0.3, $10 \mathrm{~dB}$, and $15 \mathrm{~dB}$, respectively. From the figure, we can see that the overall profit decreases as the number of users increases. It is not only because the competitions for the resource become more severe for more users, but also because more punishments are required to remove the deviation incentive.

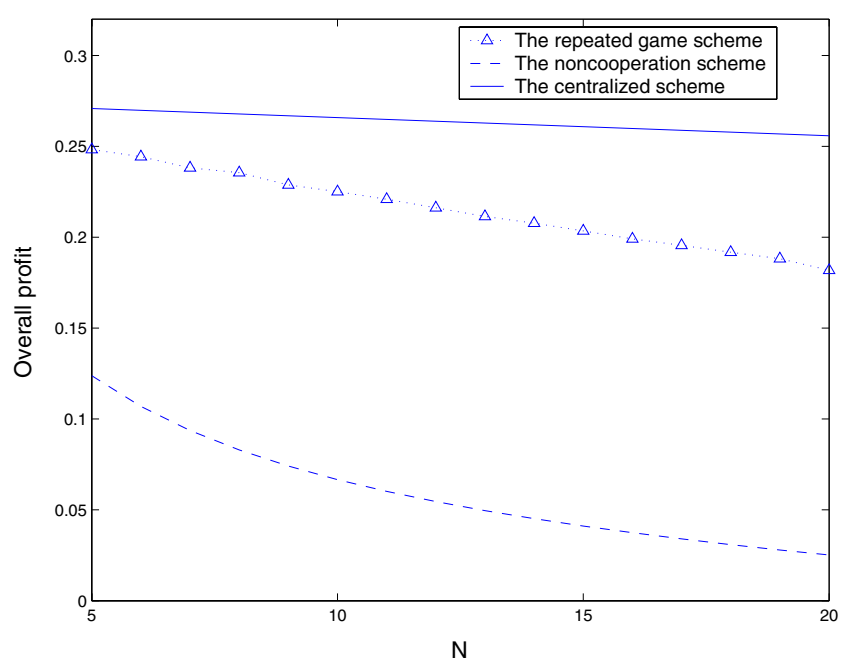

Fig. 8. Overall profit as a function of the number of users.

Moreover, considering the extreme cases, when the number of users approaches 1, the performance of our scheme is close to the that of the centralized scheme; when the number of users becomes very large, the performance of the scheme approaches that of the non-cooperative scheme. From Figure 6,7 , and 8 , the gaps between the centralized scheme and the proposed scheme are due to possible "false wars" triggered by factors such as the poor wireless channel. This gap is the cost of distributed implementation.

the average utility can be improved.

\section{COnClusions And Discussions}

In this paper, we develop a Cartel Maintenance repeated game framework to enhance collaboration among greedy users and improve the performances. In the traditional static game, the users' only concern is the current value of the utility function. These users are unable to consider the consequences of their actions in future. So the greediness can reduce the overall performances. In order to achieve cooperation among users, we construct the Cartel Maintenance repeated game framework. The benefits of cheating will be punished by the cartel formed by the other users, so that there is no incentive for any user to defect. In the proposed framework, we also develop an optimal trigger punishment game rule with the theoretical analysis.

The proposed framework is further employed to a wireless network in which users have their own authorities and centralized control is difficulty to be implemented. Each user controls its packet transmission rate so as to compete for the wireless radio resources. The proposed framework enforces cooperation among the greedy users for packet transmission in a distributed manner. As the simulation shows, the proposed scheme performs close to the centralized scheme and much better than the non-cooperative scheme, since enough punishment threat is maintained to prevent the selfish users from deviating from cooperation. 


\section{REFERENCES}

[1] D. Fudenberg and J. Tirole, Game Theory, MIT Press, Cambridge, 1991.

[2] C. Douligeris, "Multiobjective flow control in telecommunications networks," in Proc. IEEE Conference on Computer Communications (INFOCOM'92), pp. 303-312, Firenze, Italy, May 1992.

[3] T. Roughgarden and E. Tardos, "How bad is selfish routing?" in Proc. 41st Annual IEEE Symposium on the Foundations of Computer Science, Redondo Beach, CA, November 2000.

[4] C. U. Saraydar, N. B. Mandayam, and D. J. Goodman, "Pricing and power control in a multicell wireless data network," IEEE J. Select. Areas Commun., vol. 19, pp. 1883-1892, October 2001.

[5] Z. Han, Z. Ji, and K. J. R. Liu, "Power minimization for multi-cell OFDM networks using distributed non-cooperative game approach", in Proc. IEEE Global Telecommunications Conference, Dallas, TX, November 2004.

[6] S. Zhong, R. Y. Yang, and J. Chen, "Sprite: a simple, cheat-proof, creditbased system for mobile ad-hoc networks," in Proc. IEEE Conference on Computer Communications (INFOCOM'03), San Francisco, CA, March 2003.

[7] P. Michiardi and R. Molva, "A game theoretical approach to evaluate cooperation enforcement mechanisms in mobile ad-hoc networks," in Proc. International Symposium on Modeling and Optimization in Mobile, Ad Hoc, and Wireless Networks (WiOpt'03), INRIA Sophia-Antipolis, France, March 2003.

[8] Z. Han, Z. Ji, and K. J. R. Liu, "Low-complexity OFDMA channel allocation with Nash Bargaining Solution fairness", in Proc. IEEE Global Telecommunications Conference, Dallas, TX, November 2004.

[9] R. J. La and V. Anantharam, "Optimal routing control: repeated game approach", IEEE Trans. Automat. Contr., vol. 47, no. 3, pp. 437-450, March 2002.

[10] A. B. MacKenzie and S. B. Wicker, "Stability of multipacket slotted aloha with selfish users and perfect information," in Proc. IEEE Conference on Computer Communications (INFOCOM'03), San Francisco, CA, March 2003.

[11] V. Srinivasan, P. Nuggehalli, C. F. Chiasserini, and R. R. Rao, "Cooperation in wireless ad hoc networks", in Proc. IEEE Conference on Computer Communications (INFOCOM'03), San Francisco, CA, March 2003.

[12] E. Altman, A. A. Kherani, P. Michiardi, and R. Molva, "Non-cooperative forwarding in ad-hoc networks", INRIA, technique report, No. 5116, 2004.

[13] L. Anderegg and S. Eidenbenz, "Ad hoc-VCG: A truthful and cost efficient routing protocol for mobile ad hoc networks with selfish agents," ACM Annual International Conference on Mobile Computing and Networking (Mobicom'03), pp. 245-259, San Diego, CA, Sept. 2003.

[14] Z. Han, Z. Ji, and K. J. R. Liu, "Dynamic distributed rate control for wireless networks by optimal Cartel maintenance strategy", in Proc. IEEE Global Telecommunications Conference, vol.6, p.p.3454-3458, Dallas, Dec 2004

[15] R. H. Porter, "Optimal cartel trigger price strategies," J. Economic Theory, vol. 29, pp. 313-318, April 1983.

[16] D. Bertsekas and R. Gallager, Data Networks, Englewood Cliffs, N. J.: Prentice-Hall, 1992.

[17] Y. Yang and T. P. Yum, "Delay distributions of slotted ALOHA and CSMA," IEEE Trans. Commun., vol. 51, no. 11, pp. 1846-1857, November 2003.

[18] T. S. Rappaport, Wireless Communications: Principles and Practice, Prentice Hall, 1996.

[19] A. Papoulis and S. U. Pillai, Probability, Random Variables and Stochastic Processes, Fourth Edition, McGraw-Hill, 2002.

[20] S. T. Chung and A. J. Goldsmith, "Degrees of freedom in adaptive modulation: a unified view," IEEE Trans. Commun., vol. 49, no. 9, pp. 1561-1571, September 2001.

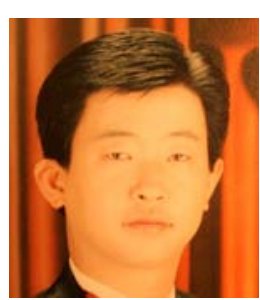

Zhu Han (S'01-M'04) received the B.S. degree in electronic engineering from Tsinghua University, in 1997, and the M.S. and Ph.D. degrees in electrical engineering from the University of Maryland, College Park, in 1999 and 2003, respectively.

From 2000 to 2002, he is an R\&D Engineer of ACTERNA, Germantown, Maryland. From 2002 to 2003, he was a Graduate Research Assistant at the University of Maryland. From 2003 to 2006, he was a Research Associate at the University of Maryland. Currently, he is an assistant Professor in Electrical and Computer Engineering Department at Boise State University, Idaho, USA. His research interests include wireless resource allocation and management, wireless communications and networking, game theory, wireless multimedia, and security.

Dr. Han is PHY/MAC Symposium vice chair of IEEE Wireless Communications and Networking Conference, 2008. Dr. Han is a member of the Technical Programming Committee for the IEEE International Conference on Communications, the IEEE Vehicular Technology Conference, the IEEE Consumer Communications and Networking Conference, the IEEE Wireless Communications and Networking Conference, and the IEEE Globe Communication Conference.

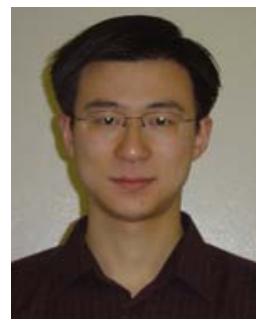

Zhu Ji received a Ph.D. degree in Electrical and Computer Engineering from University of Maryland, College Park in May 2007. He received B.S. and M.S. degrees in Electronic Engineering from Tsinghua University, Beijing, China, in 2000 and 2003, respectively. He is currently with Qualcomm, San Diego, CA. From 2003 to 2007, he was a graduate research assistant in the Communication and Signal Processing Laboratory, University of Maryland, College Park. From 2000 to 2002, he was a visiting student (research intern) in the Wireless and Networking Group at Microsoft Research Asia, Beijing, China. His research interests are in wireless communications and networking.

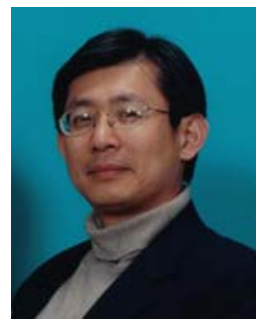

K. J. Ray Liu (F’03) is Professor and Associate Chair, Graduate Studies and Research, of Electrical and Computer Engineering Department, University of Maryland, College Park. His research contributions encompass broad aspects of wireless communications and networking, information forensics and security, multimedia communications and signal processing, bioinformatics and biomedical imaging, and signal processing algorithms and architectures.

Dr. Liu is the recipient of numerous honors and awards including best paper awards from IEEE Signal Processing Society (twice), IEEE Vehicular Technology Society, and EURASIP; IEEE Signal Processing Society Distinguished Lecturer, EURASIP Meritorious Service Award, and National Science Foundation Young Investigator Award. He also received various teaching and research recognitions from University of Maryland including Distinguished Scholar-Teacher Award, Poole and Kent Company Senior Faculty Teaching Award, and Invention of the Year Award.

Dr. Liu is Vice President - Publications and on the Board of Governor of IEEE Signal Processing Society. He was the Editor-in-Chief of IEEE Signal Processing Magazine and the founding Editor-in-Chief of EURASIP Journal on Applied Signal Processing. 